\title{
Flood Risk to the Strategic Road Network in England
}

\author{
Barry Hankin ${ }^{1, a}$, lain Craigen ${ }^{1}$, Will Rogers ${ }^{2}$, Joanne Morphet $^{3}$, Andy Bailey $^{3}$, Michael Whitehead ${ }^{3}$ \\ ${ }^{1}$ JBA Consulting, Bank Quay House, Sankey St, Warrington, WA1 1NN \\ ${ }^{2}$ AECOM, AECOM, URS House, Horne Lane, Bedford, MK40 1TS \\ ${ }^{3}$ Highways England, Woodlands, Manton Lane, Bedford, MK41 7LW
}

\begin{abstract}
It is vital that flood risks from multiple sources to the national Strategic Road Network are well understood, to help minimise disruption, reduce risk to people and to help prioritise maintenance programmes. Highways England have undertaken research to update their current understanding of risk based on improved flood mapping, plus data collected on flooding 'hot spots' through a concerted programme to record flooding events, severity and causes over the last 5 years. Building on the previous risk assessment, the roads layer was interrogated against new flood hazard data for multiple sources of flooding. The road network was divided into $100 \mathrm{~m}$ segments in order to capture a strong spatial understanding of predicted flood risk from each source, but also summarised on a $1 \mathrm{~km}$ scale and a management area level to aid prioritisation at varying scales. The $1 \mathrm{~km}$ grids were then ranked across England according to each source of flooding, and also an overall rank was derived through summing these ranks together. This was validated against detailed spatial 'hot spot' database recorded by Highways England, based on over 12,300 records, collected over 5 years.
\end{abstract}

\section{Introduction}

With an increasing number of high profile flooding events making headlines around Europe, there is a greater awareness of the cost in terms of damage, disruption and casualties. Estimates for the UK 2007 summer flooding alone put road reparation costs at $£ 190 \mathrm{~m}$, and disruption costs at $£ 98 \mathrm{~m}$, albeit highly uncertain [1]. The UK DfT recently announced emergency road reparation funds of $£ 40 \mathrm{~m}$ in the wake of UK storms Desmond and Eva for Cumbria and Lancashire.

This paper reports on an updated national screening assessment of the predicted flood risk to Highways England's Strategic Road Network (SRN) which spans all trunk roads including the slip roads to and from trunk roads within England. Figure 1 illustrates the Highways Agency Pavement Management System (HAPMS) which has been used to define the SRN across the study area. The study area shows the Highways England Management Areas (MAs).

Predicted flood risk to the SRN from each source has been scored for the carriageway split into $100 \mathrm{~m}$ segments following a technique developed for the Irish Roads Authority [2]. Each flood risk source score has then been summarised within $1 \mathrm{~km}$ grid squares as well as combining all sources to produce both weighted and unweighted overall flood risk ranks which can be used to prioritise maintenance and management. These modelled high priority risk areas were then compared against Highway England's historical Hotspot data discussed further below. This is based on approximately 12,300 individual road flooding incidents, collected by the 14 MAs since 2000, but in a systematic way for 5 years.

A suite of interactive PDF maps were produced to present the national screening assessment at both the Management Area and $1 \mathrm{~km}$ grid square scales. These are to strategically identify vulnerabilities in the national network as recommended in the European Roads Authority Network (ERANET) RIMAROCC procedure [3] and improve the prioritisation of flood risk management measures from warning to maintenance.

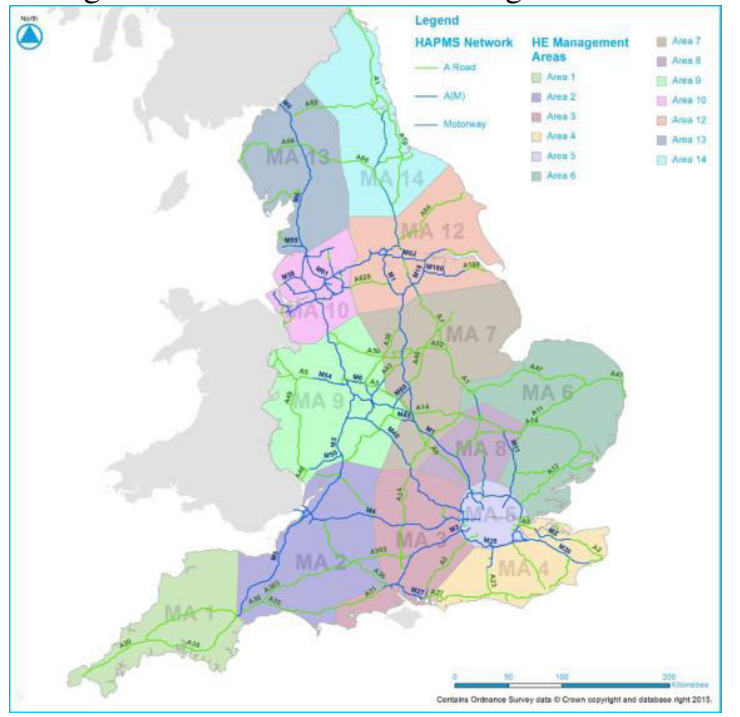

Figure 1. Highways England Strategic Road Network and Management Areas.

\footnotetext{
${ }^{a}$ Corresponding author: barry.hankin@jbaconsulting.com
} 


\section{Method}

JBA's in-house Flood Risk Metric tool (FRISM) was used to perform the GIS analysis of predicted flood risk to the SRN from each difference source. The HAPMS was split into lines no longer than $100 \mathrm{~m}$ with each segment attributed with flood risk metrics based on 4 sources of flooding and using 3 national hazard datasets:

- The Environment Agency Risk of Flooding from Rivers and Sea (RoFRS)

- The Environment Agency Updated Flood Map for Surface Water (uFMfSW)

- The BGS Areas Susceptible to Groundwater Flooding (AStGWF)

This resulted in 160,000 segments, and a high resolution dataset which was aggregated up into $1 \mathrm{~km}$ analysis units at a more manageable scale, using length weighting of the flood metrics to retain the richness of the underlying data. This resulted in approximately $8,5001 \mathrm{~km}$ grid tiles spanning the network which were then used as the spatial unit for prioritisation of risk management.

The length-weighted metric score was derived for each source and the $1 \mathrm{~km}$ tiles were ranked nationally for each flood source. These ranks were then combined to provide an overall risk ranking based on all sources of flooding. The processing steps are now discussed.

\subsection{Pre-processing the SRN}

The SRN dataset comprises a linear network with a line representing each direction of travel along the SRN. The HAPMS was pre-processed to remove oxbow lay-by features which may have skewed the identification of high flood risk areas due to an increase in the number of adjacent carriageways.

\subsection{Identifying Risk of Flooding from Rivers and Sea (RoFRS)}

This Environment Agency dataset represents the predicted flood risk originating from fluvial, tidal and coastal sources and is quantified into four probability bands summarised across $50 \times 50 \mathrm{~m}$ cells. These probabilities were generated using the Risk Assessment for Strategic Planning (RASP), whereby a systems, 'Source-Pathway-Receptor' framework [4, 5] was used to quantify the risk of defence failure and over-topping from rivers and the sea. The resulting probability bands are classified as:

- High - each year, there is a chance of flooding of greater than 1 in 30 (3.33\% Annual Exceedance Probability)

- Medium - each year, there is a chance of flooding of between 1 in $30(3.33 \%$ AEP) and 1 in 100 $(1 \%$ AEP)
- Low - each year, there is a chance of flooding of between 1 in 100 ( $1 \%$ AEP) and 1 in 1,000 $(0.1 \%$ AEP $)$

- Very Low - each year, there is a chance of flooding of less than 1 in $1,000(0.1 \%$ AEP $)$

The predicted flood risk takes into account the fragility of flood defences based on condition and construction type. The absence of any classification indicates that flood risk has not been quantified and is therefore likely to represent a very low risk. Figure 2 illustrates how the $100 \mathrm{~m}$ road segments were then attributed with the highest probability encountered along their lengths.
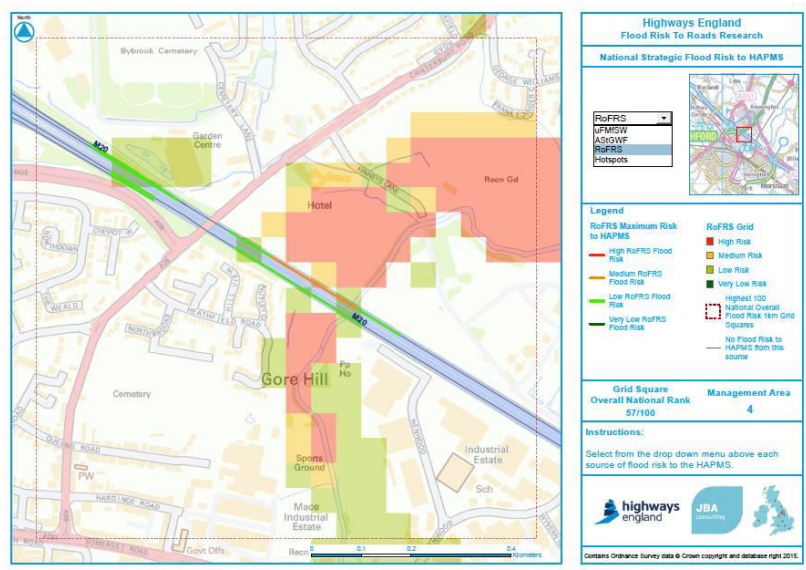

Figure 2. Road segments attributed with RoFRS

\subsection{Updated Flood Map for Surface Water (uFMfSW)}

This Environment Agency dataset represents the predicted flood risk originating from surface water/pluvial sources and is quantified into surface water depth information for three independent return periods/annual probabilities of flooding summarised across $2 \times 2 \mathrm{~m}$ cells. It was produced using the blanket rainfall approach [6], whereby different probability and duration rainfall events are applied to a DTM, and having taken into account losses using the ReFH losses model [7], and an allowance for local urban drainage, the remaining water is routed using a fast $2 \mathrm{~d}$ implementation of the 2d Shallow Water Equations [8]. The following return period events were used in this assessment, having combined the worst flooding depths across 3 different durations for each:

$$
\begin{aligned}
& \text { - } 1 \text { in } 30 \text { (3.33\% AEP) } \\
& \text { - } 1 \text { in } 100(1 \% \text { AEP }) \\
& \text { - } 1 \text { in } 1,000(0.1 \% \text { AEP })
\end{aligned}
$$

The $100 \mathrm{~m}$ road segments were attributed with the peak depth for these events, as illustrated in Figure 3 

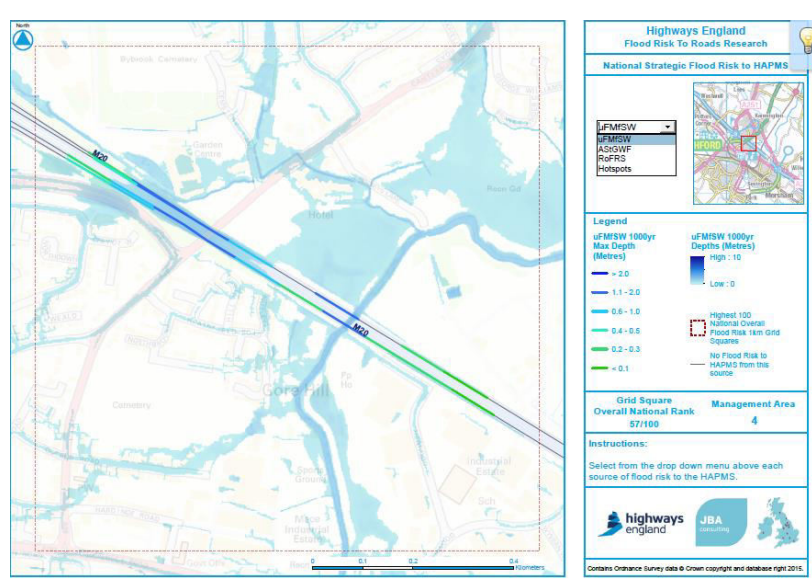

Figure 3. Attribution of the road segments with flood depth

\subsection{Areas Susceptible to Groundwater Flooding (AStGWF)}

This BGS dataset a strategic scale map showing groundwater flood areas on a $1 \mathrm{~km}$ square grid. It was developed by the Environment Agency for use by Lead Local Flood Authorities (LLFAs) for use in Preliminary Flood Risk Assessment (PFRA) as required under the Flood Risk Regulations in the UK adoption of the Floods Directive. It comprises four risk bands representing the proportion of each $1 \mathrm{~km}$ square that is susceptible to groundwater flood emergence.

$$
\begin{aligned}
& -<25 \% \\
& \bullet \geq 25 \% \text { and }<50 \% \\
& \text { - } \geq 50 \% \text { and }<75 \% \\
& \text { - } \geq 75 \%
\end{aligned}
$$

The absence of any classification indicates that flood risk has not been quantified and is therefore likely to represent a very low risk.
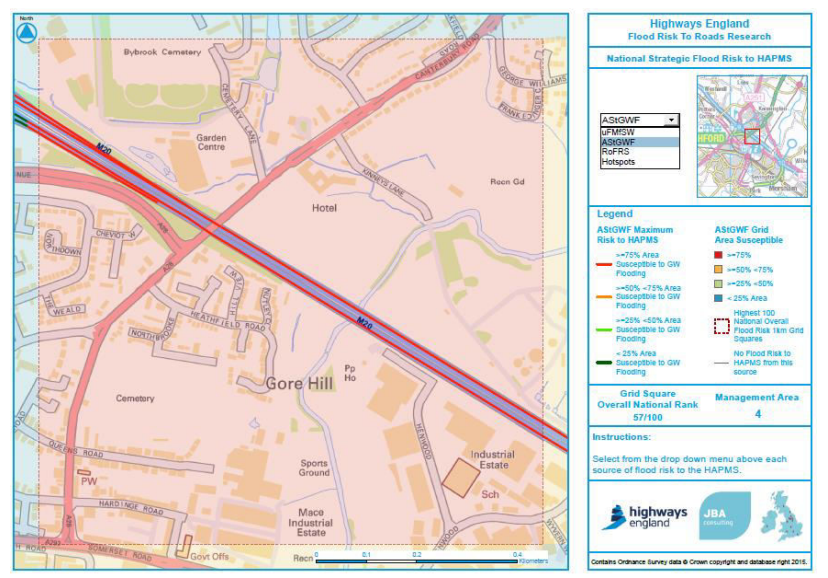

Figure 4. Road segments attributed with RoFRS

The data was pre-processed to attribute risk bands from 1 (low risk) to 4 (high risk) to the respective proportions above, so any road segments passing through a high band would be attributed with this (Figure 4).

\subsection{Adjustments to risk categories}

Using an intersection method of classifying the HAPMS with each flood risk dataset, it was observed that sections of HAPMS which rise significantly above the natural ground level were attributed as high predicted flood risk where they passed over a main watercourse. As a result, it was decided that these sections of HAPMS should be reclassified to better reflect the likelihood of the carriageway flooding.

Given the lack of SRN elevation information to identify carriageways rising above the natural ground level, a simple re-classification of flood risk was implemented as shown in Figure 5. The simple re-classification assumed that fly-overs were typically present across watercourses of a significant size only and that these watercourses would likely have been modelled by the Environment Agency and reflected within the RoFRS dataset as high flood risk. Therefore the HAPMS was intersected with the Ordnance Survey Open Rivers OpenData and the results from this intersection then buffered by $50 \mathrm{~m}$. Any segment of the HAPMS which intersected this buffer and had a RoFRS risk of high was re-attributed to low as well as re-attributing any high risk AStGWF to low risk too.

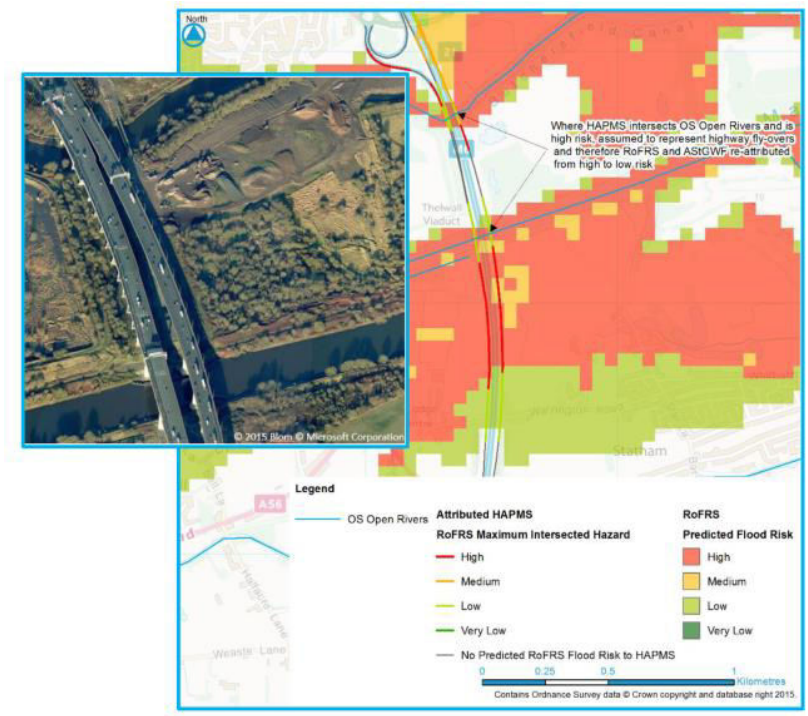

Figure 5. Correction of risk metrics

The surface water flood risk was modified to a lesser extent given that this dataset includes predicted surface water flood risk to the carriageway surface itself. However, adjustments were necessary to the uFMfSW model outputs where the topography along the HAPMS had been incised to provide the necessary hydrological continuity for the original uFMfSW. A manual check was conducted on uFMfSW predicted flood depths intersecting the HAPMS where 1000-year return period surface water maximum flood depths greater than $3.0 \mathrm{~m}$ were predicted. Sections of HAPMS where this occurred were either set to null depth (where the surrounding carriageway was not predicted at risk), or set to a representative depth (if surface water flooding to the carriageway was predicted). 


\subsection{Aggregation of risk metrics to $1 \mathrm{~km}$ unit}

Following the attribution of each HAPMS segment with the predicted flood risk from each source, the predicted flood risk was multiplied by its segment length to reflect the length of carriageway potentially at flood risk. For the surface water flood source, the three return period depth grids as a product of their segment length were equally weighted together to generate an overall surface water predicted flood risk score. An alternative method would be to use the respective probabilities to weight the depths from individual probability events, although there are different connotations of what constitutes high risk. For example, a road section that could be exposed to a rare but severe event might want to have equal importance as one that has less severe, but frequent flooding

To up-scale the predicted flood risk from the HAPMS, the predicted flood risk product score discussed above for each source of flooding was summed across all HAPMS segments within $1 \mathrm{~km}$ grid squares (Figure 6).
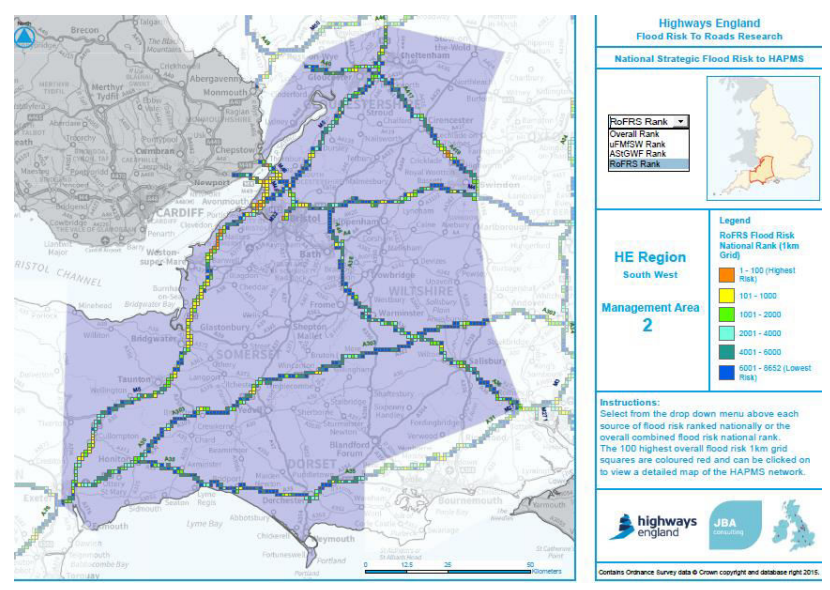

Figure 6. Aggregation to $1 \mathrm{~km}$ grid, with colour theming for 1 Management Area

The grid squares were then sorted and ranked for each source of predicted flood risk (Figure 7). In addition, an overall predicted flood risk rank was derived from summing together the ranks for individual metrics. This was implemented through both an equal/unweighted sum of each flood risk source, as well as weighted flood risk combination. The weighted combination assigned the following weights:

- $45 \%$ Surface Water (uFMfSW)

- 35\% Fluvial, Tidal and Coastal (RoFRS)

- $20 \%$ Groundwater (AStGWF)

\section{Results}

The results from this ranking analysis were used to identify the worst 100 grid squares in terms of overall flood risk to the HAPMS, as shown in Figure 6 for one Management Area. This was undertaken twice, once with the same weights for different sources, and another time with the above weights.
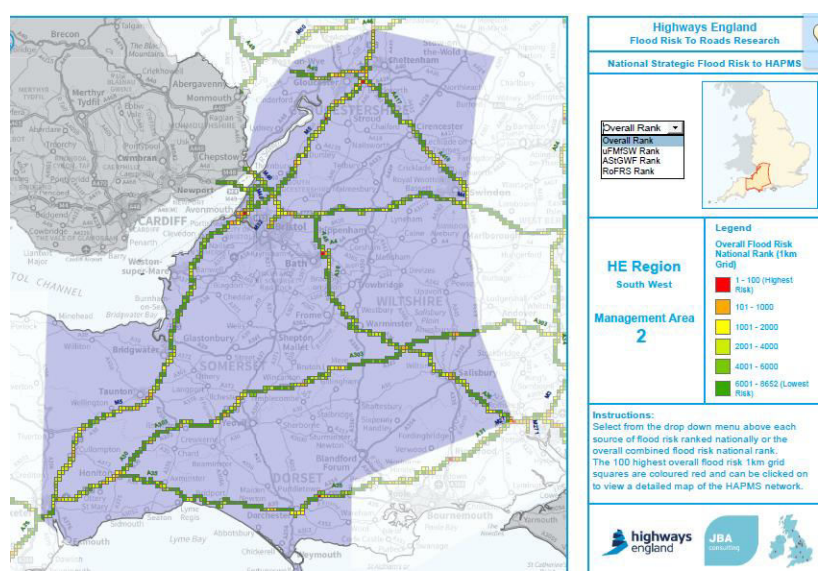

Figure 7. Overall risk ranking across all sources

The results from the strategic national screening assessment are best viewed via the suite of interactive PDF maps (see schematic Figure 8). The individual sources of flood risk or combined overall flood risk ranks can be viewed for $1 \mathrm{~km}$ grid squares displayed on the MA scale maps. More detailed flood risk information to the individual HAPMS segments are displayed on the detailed maps for the top 100 highest overall (unweighted) flood risk ranks.

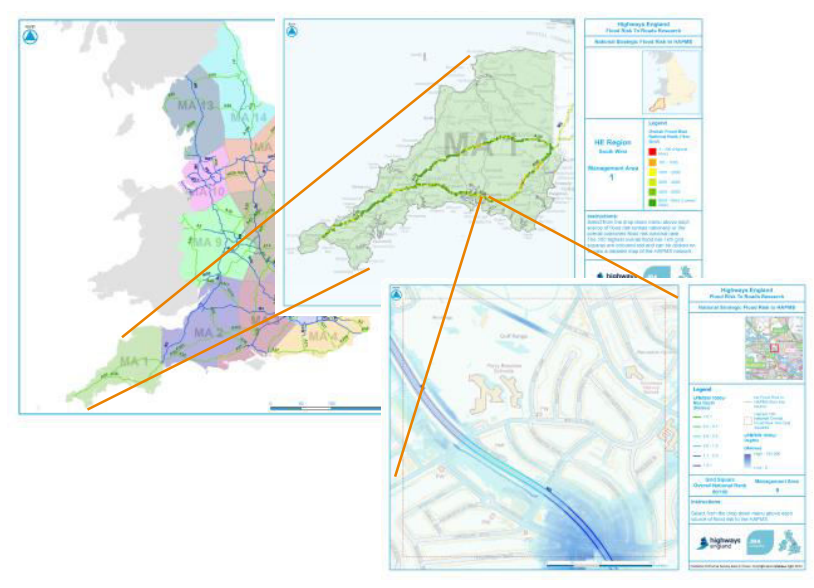

Figure 8. Overall risk ranking across all sources 


\section{Validation}

The areas of greatest predicted flood risk identified in the national screening assessment above were then compared against Highways England's historic hotspot dataset. This provides a record of the number of known flood events which have impacted the SRN over the last 5 years. Each hotspot is defined for a particular carriageway direction, and Figure 9 shows how Management Areas identify the hotspots on the HADDMS system.
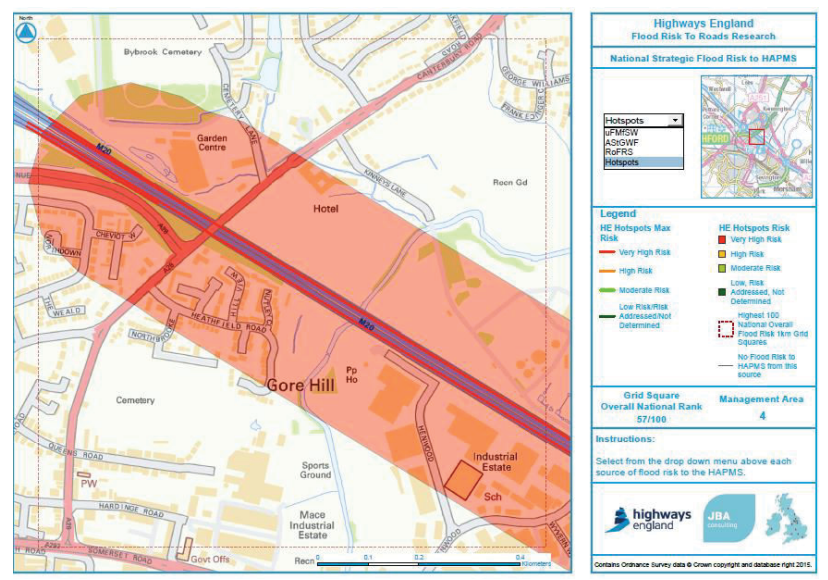

Figure 9. Overall risk ranking across all sources

These hotspots comprise a range of overall risk statuses: very high; high; moderate; low, risk assessed; or not determined as shown in the risk matrix in Figure 10. This overall risk rating based on a combination of frequency of observed flooding issues (horizontal ) and a flood severity index, FSI (on vertical axis). If the observed flooding is outside an Environment Agency flood risk zone, then the risk is determined as higher for Highways England as the source is more likely to be as a result of the failure of its own drainage assets.

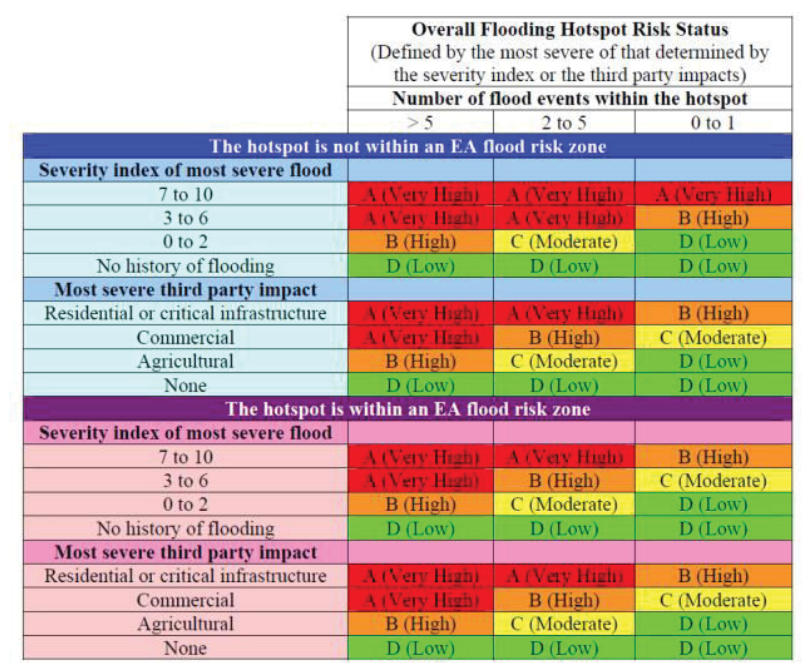

Figure 10. Highways England hotspots overall flood risk status classification based on frequency and severity matrix
The FSI is based on a product of 4 factors, based on road type, traffic volume, extent of carriageway blocked, and duration of blocked carriageway. This is normalised on a score of 1 to 10 , with 10 corresponding to severe delays on a busy motorway with all carriages blocked.

The validation involved assessing the overlap or capture of the hotspots with worst $1 \mathrm{~km}$ grid squares as based on the predicted flood risk. It was found that approximately half $(50 \%)$ of the worst 100 grid squares (based on the national assessment) captured at least 1 hot spot.

However, there are a large number of historic hotspots, and the top 100 unweighted grid squares only represent $8 \%$ of these. A more generic curve was generated to understand the proportion of hot-spots captured depending on the rank of the $1 \mathrm{~km}$ grid squares in Figure 11. This shows how the top 1,000 unweighted grid squares represent $44 \%$ of all hotspots (orange curve).

The hotspots were based on a local Area assessment of the frequency of flooding and flood severity, and a spatial aggregation to a road intersection. Therefore the underlying historic flooding point data was also assessed in terms of capture. The light blue curve shows the capture of the historic records based on the highest FSI (greater than 6 in Figure 10). This is steeper than the more generic capture of the spatial hotspots, and shows that the higher ranked $1 \mathrm{~km}$ squares based on the predictive modelling are more discerning.

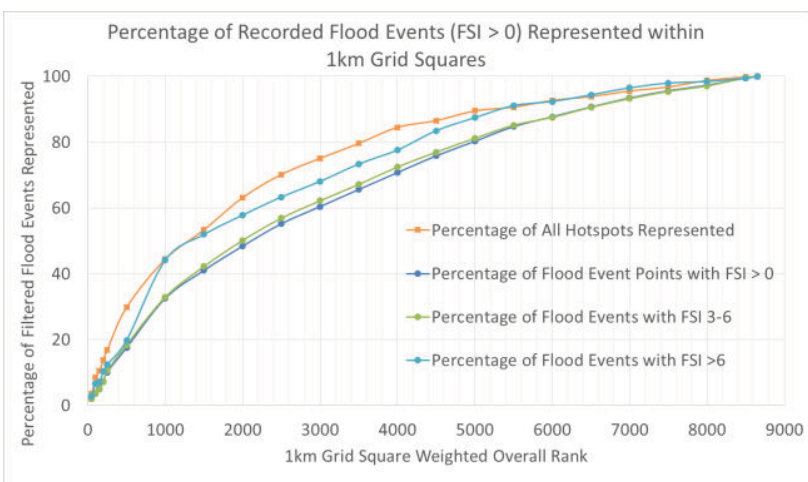

Figure 11. Overall risk ranking across all sources

It is important to consider that the Highways England hotspot dataset provides a relatively short (five years) record of observed flood events, whilst the predicted flood risk datasets used to screen the SRN typically relate to significantly rarer probabilistic events. For example, a 1 in 30 year return period event is unlikely to have been captured by a 5 year record.

\section{Discussion}

The validation of the national flood risk assessment based on four sources of flooding against historic data is promising, but does require further validation as the historic record length grows. There are new datasets becoming available that can be used to improve the 
assessment, but given the degree of agreement it would now be worth using the assessment to help prioritise maintenance, or possibly risk management measures such as issuing flood warning to drivers. The suite of interactive maps enables rapid appraisal of these high risk areas, as the user can be taken from a national overview to a detailed $1 \mathrm{~km}^{2}$ level of detail for the higher risk areas in two clicks of a mouse. They can then assess the key sources of flood risk, and make a more detailed investigation of a risk management measure. For those records where a cause of flooding was identified. Since over $50 \%$ were attributed to blockage of drains/gullies, so the prioritisation of maintenance measures would be a key recommendation.

The detailed flood risk data that has been attributed to the SRN can also be used for a number of risk management activities:

- Combined with traffic data (for example Annual Average Daily Traffic) to estimate traffic disruption and potentially costs. These are very uncertain, but could be important for soliciting partnership funding from for example businesses that are impacted the most.

-Combined with a loss function, the percentage of the SSRN at risk can be defined. For example, the Flood Risk to People study [9], an average saloon car can be floated for depths above $0.25 \mathrm{~m}$ and velocities $>1.5 \mathrm{~m} / \mathrm{s}$. Without considering velocities, the analysis shows that for the 1 in 30 year Return Period, 5\% of the network could meet this criteria. Combined with high risk of flooding from rivers or sea, this increases to $8 \%$. However, these figures are indicative for a particular probability event and have not been quantified in terms of long term annual average risk. Furthermore, the surface water modelling effectively assumes that the drainage infrastructure is blocked unless in an urban area, and more modelling would be recommended

- The understanding of the potential exposure of the SRN could now be combined with a probabilistic handling of drainage failures. This would require an analysis of the historic flooding records, to identify the relationship between the probability of the rainfall event giving rise to different severity blockage events. This could be made contingent on explanatory variables such as 'time since last maintenance visit'.

-The information could also be combined with flooding from blockage of culverts. In a separate study (not reported) the risk to third parties from the blockage of culverts was assessed through modelling the damages avoided through maintaining all assets to avoid blockage. Currently the risk to the road network and users is assessed separately to the risk to third parties, although the historic hotspots do take this into account for recorded flooding.

\section{Conclusions and Recommendations}

The strategic national screening assessment has investigated the predicted flood risk from fluvial, coastal, tidal, groundwater and surface water sources to the Highways England Strategic Road Network (SRN). Predicted flood risk to the SRN from each source has been scored for the carriageway split into approximately 160,000 segments of $100 \mathrm{~m}$ length or less. Each flood risk source score has then been summarised within $1 \mathrm{~km}$ grid squares as well as combining all sources to produce both weighted and unweighted overall flood risk ranks to signpost areas at greatest risk across all sources investigated. A suite of interactive PDF maps have been produced to present the national screening assessment at both the Management Area and $1 \mathrm{~km}$ grid square scales. The results from this analysis are aimed to strategically identify high risk flood locations within the national network to improve the prioritisation of flood risk management.

The following are recommended:

-At the time of the national screening assessment implementation, there was no freely available high resolution LiDAR elevation dataset for determining carriageway elevation information. As a result, a simplified approach to identifying carriageway fly-overs and re-classification of flood risk to the SRN was adopted. Since September 2015, the Environment Agency have released their existing LiDAR data repository under an Open Government Licence freely permitting the use of this high resolution elevation dataset for both commercial and noncommercial purposes. It is recommended that future work aims to attribute the Highways England SRN with elevation information to improve the filtering of datasets at carriageway fly-overs to minimise any overestimation of predicted flood risk attribution.

-Whilst the national strategic assessment is useful to understand parts of the SRN at risk from different sources of flooding, and useful for planning and maintenance activities, it is ultimately a static risk assessment. With improvements in rainfall forecasting and telemetered river data, it is becoming more practical to generate a real-time flood footprint to help with emergency response and incident management. Improvements in meteorological forecasting would permit a Dynamic assessment with warning

-The historic flooding data should be combined with information on the rarity of the rainfall and used to construct a drainage failure model to help understand the probability of failure through gully blockage. 


\section{References}

1. Chatterton JB, Viavattene C, Morris J, PenningRowsell EC and Tapsell S (2010) The Costs of the Summer 2007 Floods in England. Project: SC070039/R1. Environment Agency, Bristol.

2. Hankin, B., Bryant R., O’Malley, V., Kitchen, A., and Dobson, R. (2014) A Flood Risk Management Protocol for Roads. Infrastructure Asset Management, ICE. http://dx.doi.org/10.1680/iasma.14.00017. Paper 1400017

3. ERA-NET ROAD (2010) Risk Management for Roads in a Changing Climate: $A$ Guidebook to the RIMAROCC Method. Project No. TR80A 2008:72148.

4. HALL J.W., DAWSON R.J., SAYERS, P., ROSU, C., CHATTERTON, J., DEAKIN, R. 2003. A methodology for national-scale flood risk assessment. Proceedings of the Institution of Civil Engineers - Water \& Maritime Engineering 2003, 156(3), 235-247.

5. ENVIRONMENT AGENCY (2005). Risk assessment of flood and coastal defence systems for strategic planning (RASP). ISBN 1844323722, LIT 1967. (https://www.gov.uk/government/publications/riskassessment-of-flood-and-coastal-defence-systemsfor-strategic-planning-rasp)

6. Hankin B, Waller S, Astle G and Kellagher R (2008). Mapping space for water: screening for urban flash flooding. Journal of Flood Risk Management 1(1): 13-22.

7. Kjeldson, T., (2007). The Revitalised FSR/FEH rainfall-runoff method. Centre for Ecology and Hydrology (ISBN 0903741 157)

8. Lamb R, Crossley A and Waller S (2009) A fast 2D floodplain inundation model. Proceedings of the Institution of Civil Engineers: Water Management 162.

9. DEFRA (2005). Flood Risks to People, Phase 2, FD2321/TR1, The Flood Risks to People Methodology. Flood and Coastal R\&D Programme. London: Defra 